\title{
TRANSITIVITY AND THE $\boldsymbol{\gamma}$-SPACE CONJECTURE IN ORDERED SPACES
}

\author{
JACOB KOFNER
}

\begin{abstract}
Each generalized ordered $\gamma$-space is nonarchimedean quasimetrizable. Moreover, each generalized ordered space is 3-transitive, i.e. for each neighbournet $U$ there is a transitive neighbournet $V \subset U^{3}$.
\end{abstract}

\section{Introduction.}

1. The concept of a quasimetric is quite old [N1], [W]. It is a generalized metric $d$ with the triangle axiom $d(x, z) \leqslant d(x, y)+d(y, z)$, but generally without the symmetry axiom $d(x, y)=d(y, x)$. If the triangle axiom is strengthened to " $d(x, y)$ $<\max \{d(x, y), d(y, z)\} "$, then $d$ is a nonarchimedean quasimetric [FL1], [K]. If it is relaxed to " $d\left(x, y_{n}\right) \rightarrow 0$ and $d\left(y_{n}, z_{n}\right) \rightarrow 0$ implies $d\left(x, z_{n}\right)$ ", then $d$ is a $\gamma$-metric and a space with a $\gamma$-metric is a $\gamma$-space [N2], [H].

Obviously, a nonarchimedean quasimetric space is quasimetric and a quasimetric space is a $\gamma$-space. However, a quasimetric space need not be nonarchimedean quasimetrizable [K], while the problem whether each $\gamma$-space is quasi-metrizable, listed as Classic Problem VIII in [T], is open and only partial solutions have been obtained [G], [J1], [B], [F]. In [B] it was shown that if a linearly ordered space $X$ has a $\sigma$-discrete dense set then every generalized ordered space constructed on $X$ is quasimetrizable if it is a $\gamma$-space.

It will be proved here that each generalized ordered $\gamma$-space is nonarchimedean quasimetrizable.

2. That result will follow from more general results concerned with the transitivity of ordered spaces.

Recall that a binary relation on a set $X$ is any subset $R$ of $X \times X$. Given a binary relation $R$ on $X$ we define $R\{x\}=\{y \in X \mid(x, y) \in R\}$. Conversely, we can construct a binary relation on $X$ by specifying each of the sets $R\{x\}$ and then letting $R=\cup\{\{x\} \times R\{x\} \mid x \in X\}$. Throughout the rest of this paper, we will systematically confuse binary relations as subsets of $X^{2}$ and binary relations which are obtained by specifying subsets $R\{x\}$ of $X$, as before. For a binary relation $R$ on $X$ and any subset $G \subset X$, we define $R G=R(G)=\cup\{R\{x\} \mid x \in G\}$. Given two binary relations $R$ and $S$ on $X$, a new binary relation $S \circ R$ is defined by $S \circ R=\{(x, z) \mid$ for some $y \in X,(x, y) \in R$ and $(y, z) \in S\}$. Equivalently, $S \circ R$ can be obtained by defining $(S \circ R)\{x\}=S(R\{x\})$. If $R$ is a binary relation, then

Received by the editors December 20, 1979 and, in revised form, April 22, 1980.

1980 Mathematics Subject Classification. Primary 54E15; Secondary 54F05, 54G20.

Key words and phrases. Quasimetric, nonarchimedean, $\gamma$-space, ordered space, transitive space, neighbournet. 
$R^{2}$ denotes $R \circ R, R^{3}=R \circ R^{2}$, etc. A binary relation is transitive if $R^{2} \subset R$, i.e., if $z \in R\{x\}$ whenever $z \in R\{y\}$ and $y \in R\{x\}$.

A binary relation $U$ on a space $X$ is called neighbournet in $X$ provided that each $U\{x\}$ is a neighbourhood of $x$. If $U$ is a neighbourhood of the diagonal in $X \times X$, then it is a neighbournet in $X$, but the converse need not be right.

A sequence $\left\langle U_{n}\right\rangle$ of neighbournets is called basic if for each $x \in X,\left\{U_{n}\{x\} \mid\right.$ $n \geqslant 1\}$ is a neighbourhood base at $x$, and the sequence is called a normal sequence if $U_{n+1}^{2} \subset U_{n}$ for each $n$. A neighbournet $U$ is said to be normal if it is a member of a normal sequence of neighbournets [J2]. Clearly, if $d$ is a quasimetric for $X$ and if $U_{n}=\left\{(x, y) \in X^{2} \mid d(x, y)<1 / 2^{n}\right\}$, then $\left\langle U_{n}\right\rangle$ is a basic, normal sequence. Indeed, the following characterization is known [FL1], [K], [LF], [J2].

Proposition 1. (a) $A$ space $X$ is quasimetrizable if and only if $X$ has a basic, normal sequence of neighbournets.

(b) $A$ space $X$ is nonarchimedean quasimetrizable if and only if $X$ has a basic sequence of transitive neighbournets.

(c) A space $X$ is a $\gamma$-space if and only if there is a sequence $\left\langle U_{n}\right\rangle$ of neighbournets in $X$ such that $\left\langle U_{n}^{2}\right\rangle$ is a basic sequence.

Concerning (b) of Proposition 1, we remark that any decreasing sequence of transitive neighbournets is a normal sequence; in part (c), if $\left\langle U_{n}^{2}\right\rangle$ is basic, then so is $\left\langle U_{n}^{k}\right\rangle$ for each fixed $\left.k\right\rangle 1$.

A $\gamma$-space $X$ is quasimetrizable (resp., nonarchimedean quasimetrizable) if for some $k \geqslant 1$ and for each neighbournet $U$, there is a normal (resp. transitive) neighbournet $V$ with $V \subset U^{k}$.

A space is called transitive if for each normal neighbournet $U$ there is a transitive neighbournet $V \subset U$, and is $k$-transitive if for each neighbournet $U$ there is a transitive neighbournet $V \subset U^{k}$ [FL2], [FL3]. It follows that $k$-transitive $\gamma$-spaces $(k>1)$ as well as transitive quasimetric spaces are nonarchimedean quasimetrizable.

Obviously, a $k$-transitive space is transitive as well as $(k+1)$-transitive. A space is seldom $k$-transitive (cf. later Remark). Quasimetric spaces which are not nonarchimedean quasimetrizable fail to be transitive. Metric spaces are transitive [J2]. These and other results on transitive spaces can be found in [J2], [FL3]. It is not known, however, if other common types of spaces, for example, compact spaces or nonarchimedean quasimetrizable spaces, are transitive.

The main result of this paper states that all generalized ordered spaces are 3-transitive.

3. A Hausdorff space is called generalized ordered space (= GO-space) if the underlying set is linearly ordered and some collection of intervals forms a base for the topology; i.e. for each $x \in X$ either all ] $a, b$, with $a<x<b$; or all ] $a$, $x$ ], with $a<x$; or all [ $x, b$ [, with $x<b$; or $\{x\}$ form a base of neighbourhoods [Lu].

Let $X$ be a space, and $U$ be a neighbournet in $X$. Define a new neighbournet $U^{+}$ by setting $U^{+}\{x\}=\cap\{U G \mid G$ is a neighbourhood of $x\}$. One has $U \subset U^{+} \subset$ $U V$ for each neighbournet $V$ in $X$, and hence $U^{k} \subset\left(U^{k}\right)^{+} \subset U^{k+1}$ for each $k>1$. If $G$ is an open set then $U^{+}(G)=U(G)$. 
II. 2-transitivity in Sorgenfrey spaces. A GO-space is called Sorgenfrey if each [ $x, \rightarrow$ [ is open in $X$. Let $X$ be a Sorgenfrey space and let $U$ be a neighbournet in $X$. Given a set $Y \subset X$ and a point $r \in X$ we put $R(Y, r, U)=\{x<r \| x, r[\subset Y \cap$ $\left.U^{+}\{x\}\right\}$. A family $\left\langle\left\langle R_{\alpha}, r_{\alpha}\right\rangle\right\rangle_{\alpha \in A}$, where $A$ is an initial set of ordinals, is called $U$-regular in $X$ provided that $R_{\alpha}=R\left(X-\cup_{\beta<\alpha} R_{\beta}, r_{\alpha}, U\right)$ for each $\alpha$.

LEMMA 1. Let $X$ be a Sorgenfrey space, $U$ be a neighbournet and $\left\langle\left\langle R_{\alpha}, r_{\alpha}\right\rangle\right\rangle_{\alpha<\alpha_{0}}$ be a $U$-regular family. Then

(a) the sets $R_{\alpha}$ are disjoint,

(b) if $x \in R_{\alpha}$ then $\left[x, r_{\alpha}\left[\subset U^{+}\{x\}\right.\right.$,

(c) if $x \in R_{\alpha}, y \in R_{\alpha^{\prime}}$ and $y \in\left[x, r_{\alpha}\left[\right.\right.$ then $\alpha^{\prime}>\alpha$ and $r_{\alpha^{\prime}}<r_{\alpha}$,

(d) for each $\alpha \leqslant \alpha_{0}$ the set $\cup_{\beta<\alpha} R_{\alpha}$ is closed in $X$.

Proof. (a) and (b) are obvious.

(c) Since $y \in\left[x, r_{\alpha}\left[\subset X-\cup_{\beta<\alpha} R_{\beta}\right.\right.$ we get $\alpha^{\prime}>\alpha$. If $r_{\alpha^{\prime}}>r_{\alpha}$ we have $\left[y, r_{\alpha}[\subset\right.$ $\left[y, r_{\alpha^{\prime}}\left[\subset U^{+}\{y\}\right.\right.$ and $\left[y, r_{\alpha}\left[\subset\left[x, r_{\alpha}\left[\subset X-\cup_{\beta<\alpha} R_{\beta}\right.\right.\right.\right.$; hence $y \in R_{\alpha}$, i.e. $\alpha^{\prime}=\alpha$.

(d) Suppose that $\alpha$ is the smallest ordinal $<\alpha_{0}$ such that $\cup_{\beta<\alpha} R_{\beta}$ is not closed and let $x \in \operatorname{cl}\left(\cup_{\beta<\alpha} R_{\beta}\right)-\cup_{\beta<\alpha} R_{\beta}$.

If $\alpha=\beta+1$, then $\operatorname{cl} \cup_{\beta<\alpha} R_{\beta}=\operatorname{cl}\left(\cup_{\gamma<\beta} R_{\gamma}\right) \cup \operatorname{cl~} R_{\beta}=\cup_{\gamma<\beta} R_{\gamma} \cup \operatorname{cl} R_{\beta}$. The set $\cup_{\beta<\gamma} R_{\gamma}$ is closed and hence $x \in \operatorname{cl} R_{\beta}$. Let us show that $x \in R_{\beta}$. Indeed, if $G$ is an open neighbourhood of $x$ and $G \subset\left[x, \rightarrow[\right.$ then one has $] x, r_{\beta}[\subset$ $\cup\left\{\left[y, r_{\beta}\left[\mid y \in G \cap R_{\beta}\right\} \subset \cup\left\{\left[y, r_{\beta}\left[\mid y \in R_{\beta}\right\} \subset X-\cup_{\gamma<\beta} R_{\gamma}\right.\right.\right.\right.$ by definition of $R_{\beta}$. On the other hand, by the same definition,

$$
\bigcup\left\{\left[y, r_{\beta}\right] \mid y \in G \cap R_{\beta}\right\} \subset U^{+}\left(G \cap R_{\beta}\right) \subset U^{+}(G)=U(G)
$$

since $G$ is an open set. Hence

$$
\begin{aligned}
{\left[x, r_{\beta}\left[\subset\left(X-\bigcup_{\gamma<\beta} R_{\beta}\right) \cap(\cap\{U(G) \mid G \text { is a neighbourhood of } x\})\right.\right.} \\
\subset\left(X-\bigcup_{\gamma<\beta} R_{\gamma}\right) \cap U^{+}\{x\},
\end{aligned}
$$

i.e. $x \in R\left(X-\cup_{\gamma<\beta} R_{\gamma}, r_{\beta}, U\right)=R_{x_{\beta}}$. This contradicts $x \notin \cup_{\gamma<\alpha} R_{\gamma}$.

If $\alpha=\sup \{\beta \mid \beta<\alpha\}$, we pick $y \in R_{\alpha^{\prime}}$ with $\alpha^{\prime}<\alpha,\left[x, y\left[\subset U\{x\}\right.\right.$, and $z \in R_{\beta}$ with the smallest $\beta, \alpha^{\prime}<\beta<\alpha$ such that $\left[x, z\left[\subset X-\cup_{\gamma<\alpha} R\right.\right.$. Obviously [x, $z$ [ $\subset X-\cup_{\gamma<\beta} R_{\gamma}$. Since $y \in R_{\alpha^{\prime}} \subset \cup_{\gamma<\beta} R_{\gamma}$ we have $y \notin[x, z[$. We also have $y \in\left[z, r_{\beta}\left[\right.\right.$ by (c) since $z \in R_{\beta}, y \in R_{\alpha}$ and $\alpha^{\prime}<\beta$. Hence $\left[x, r_{\beta}[\subset[x, y[\subset U\{x\}\right.$ and $\left[x, r_{\beta}\left[=\left[x, z\left[\cup\left[z, r_{\beta}\left[\subset X-\cup_{\gamma<\beta} R_{\gamma}\right.\right.\right.\right.\right.\right.$. Hence

$$
x \in R\left(X-\bigcup_{\gamma<\beta} R_{\gamma}, r_{\beta}, U\right)=R_{\beta}
$$

and this contradicts $x \notin \cup_{\gamma<\alpha} R_{\gamma}$.

Set $R_{U}=\left\{x \mid \exists r>x,\left[x, r\left[\subset U^{+}\{x\}\right\}\right.\right.$. Each $R(Y, r, U) \subset R_{U}$ hence if $\left\langle R_{\alpha}, r_{\alpha}\right\rangle$ is a $U$-regular family then $\cup_{\alpha} R_{\alpha} \subset R_{U}$. A $U$-regular family $\left\langle\left\langle R_{\alpha}, r_{\alpha}\right\rangle\right\rangle$ is called maximal if $\cup_{\alpha} R_{\alpha}=R_{U}$.

LEMMA 2. For each Sorgenfrey space $X$ and neighbournet $U$ in $X$ there exists a maximal $U$-regular family in $X$. 
PROof. Suppose that we already have a regular family $\left\langle\left\langle R_{\alpha}, r_{\alpha}\right\rangle\right\rangle$ and $\cup_{\beta<\alpha} R_{\beta}$ $\neq R_{U}$. Extend the family as follows. Let $x \in R_{U}-\cup_{\beta<\alpha} R_{\beta}$. The set $\cup_{\beta<\alpha} R_{\beta}$ is closed by Lemma 1(d). Hence for some $r_{\alpha} \in X$ we have

$$
\left[x, r_{\alpha}\left[\subset U\{x\} \cap\left(X-\bigcup_{\beta<\alpha} R_{\beta}\right)\right. \text {. }\right.
$$

Then $R_{\alpha}=R\left(X-\cup_{\beta<\alpha} R_{\beta}, r_{\alpha}, U\right) \neq \varnothing$ since $x \in R_{\alpha}$.

THEOREM 1. In a Sorgenfrey space, for each neighbournet $U$ there exists a transitive neighbournet $V \subset U^{+}$.

Proof. Let $\left\langle\left\langle R_{\alpha}, r_{\alpha}\right\rangle\right\rangle$ be a maximal $U$-regular family in $X$ by Lemma 2 . For $x \in X-R_{U}$ set $V\{x\}=\{x\}$. By Lemma $1(\mathrm{a}),\left\langle R_{\alpha}\right\rangle$ is a partition of $\boldsymbol{R}_{U}$. For $x \in R_{\alpha}$ set $V\{x\}=\left[x, r_{\alpha}\left[\right.\right.$. Then $V\{x\} \subset U^{+}\{x\}$ by Lemma 1(b). If $x \in R_{\alpha}$ and $y \in R_{\alpha^{\prime}}, y \in V\{x\} \subset\left[x, r_{\alpha}\left[\right.\right.$; then by Lemma $1(\mathrm{c}) r_{\alpha^{\prime}} \leqslant r_{\alpha}$ and $V\{y\}=\left[y, r_{\alpha}^{\prime}[\subset\right.$ $\left[x, r_{\alpha}[=V\{x\}\right.$; hence $V$ is transitive.

Since $U^{+} \subset U^{2}$ we get

THEOREM 2. Each Sorgenfrey space is 2-transitive.

III. 3-transitivity in GO-spaces. Let $X$ be a GO-space. Associate with $X$ two spaces $X_{r}$ and $X_{l}$ on the underlying set of $X$. The topology of $X_{r}\left(X_{l}\right)$ is a supremum of the topology of $X$ and that generated by all intervals $[x, y[(] x, y]) . X_{r}$ is a Sorgenfrey space with respect to the order of $X$ and $X_{l}$ is a Sorgenfrey space with respect to the opposite order.

Let $U$ be a neighbournet in $X$. Set $U_{r}\{x\}=U\{x\} \cap\left[x, \rightarrow\left[\right.\right.$ and $U_{l}\{x\}=U\{x\}$ $\cap] \rightarrow, x$ ]. Then $U_{r}\left(U_{l}\right)$ is a neighbournet in $X_{r}\left(X_{l}\right)$.

Proposition 2. Let $X$ be a GO-space and let $U$ be a neighbournet in $X$ such that for some neighbournet $W, U=W^{2}$ where $W_{r}$ and $W_{l}$ are transitive neighbournets in $X_{r}$ and $X_{l}$. Then there exists a transitive neighbournet $V \subset U^{+}$.

Proof. If we define a transitive neighbournet $V^{R}$ in $X$ such that $V_{r}^{R} \subset U^{+}$and, similarly dealing with the opposite order, a transitive neighbournet $V^{L}$ in $X$ such that $V_{l}^{L} \subset U^{+}$, we shall set $V=V^{L} \cap V^{R}$. Then $V$ will be a transitive neighbournet in $X$ and $V=V_{l} \cup V_{r} \subset V^{L} \cup V_{r}^{R} \subset U^{+}$, and the proof will be completed.

So let us define $V^{R}$.

Note that $U$, which is a neighbournet in $X$, can be considered as a neighbournet in $X_{r}$. Apply the operation ${ }^{+}$to $U$ in $X_{r}$. Denote the result by $U_{X_{r}}^{+}$. We have $U_{X_{r}}^{+} \subset U^{+}$. The space $X_{r}$ is a Sorgenfrey space and hence by Lemma 2 there exists a maximal $U$-regular family $\left\langle\left\langle R_{\alpha}, r_{\alpha}\right\rangle\right\rangle$ in $X_{r}$.

Set $\tilde{V}\{x\}=] \leftarrow, x]$ when $x \notin R_{U}$ and $\left.\tilde{V}\{x\}=\right] \leftarrow, r_{\alpha}\left[\right.$ when $x \in R_{\alpha}$, and $P(x)=\{y \in \tilde{V}\{x\} \mid \tilde{V}\{y\} \not \subset \tilde{V}\{x\}\}$.

Now define $V^{R}\{x\}=\tilde{V}\{x\}-P(x)$. Since $y \in V^{R}\{x\}$ if and only if $\tilde{V}\{y\} \subset$ $\tilde{V}\{x\}, V^{R}$ is transitive.

$V_{r}^{R}\{x\}$ is either $\{x\}$ or for $x \in R_{\alpha}, V_{r}^{R}\{x\} \subset\left[x, r_{\alpha}\right] \subset U_{X_{r}}^{+}\{x\} \subset U^{+}\{x\}$. 
It remains to show that $V^{R}$ is a neighbournet in $X$. We will use the fact that $U=W^{2}$, where $W$ is a neighbournet in $X$ such that $W_{l}$ and $W_{r}$ are transitive. We shall need the following notes.

$$
\left(W^{2}\right)_{r}=\left(W \circ W_{l}\right)_{r}
$$

Indeed $\left(W^{2}\right)_{r}=\left(W \circ\left(W_{l} \cup W_{r}\right)\right)_{r}=\left(W \circ W_{l}\right)_{r} \cup\left(W \circ W_{r}\right)_{r}=\left(W \circ W_{l}\right)_{r} \cup$ $\left(\left(W_{l} \cup W_{r}\right) \circ W_{r}\right)_{r}=\left(W \circ W_{l}\right)_{r} \cup\left(W_{l} \circ W_{r}\right)_{r} \cup\left(W_{r}^{2}\right)=\left(W \circ W_{l}\right)_{r} \cup W_{r} \cup W_{r}$ since $\left(W_{l} \circ W_{r}\right)_{r}=W_{r}$ and $W_{r}$ is transitive.

$$
\begin{aligned}
& \text { If } G \subset X, x \in X \text { and for each } y \in G, y<x \text { then } W^{2}(G) \cap \\
& {\left[x, \rightarrow\left[=\left(\left(W \circ W_{l}\right)(G)\right) \cap[x, \rightarrow[.\right.\right.}
\end{aligned}
$$

Indeed $W^{2}(G) \cap\left[x, \rightarrow\left[=\left(\cup_{y \in G} W^{2}\{y\}\right) \cap\left[x, \rightarrow\left[=\left(\cup_{y \in G} W^{2}\{y\} \cap\right.\right.\right.\right.\right.$ $\left[y, \rightarrow[) \cap\left[x, \rightarrow\left[=\left(\cup_{y \in G}\left(W^{2}\right)_{r}\{y\}\right) \cap\left[x, \rightarrow\left[=\left(W^{2}\right)_{r}(G) \cap[x, \rightarrow[\right.\right.\right.\right.\right.$. By (i) the last set is $\left(W \circ W_{l}\right)_{r}(G) \cap\left[x, \rightarrow\left[=\left(\cup_{y \in G}\left(W \circ W_{l}\right)\{y\} \cap[y, \rightarrow[) \cap[x, \rightarrow[=\right.\right.\right.$ $\left(\cup_{y \in G}\left(W \circ W_{l}\right)\{y\}\right) \cap\left[x, \rightarrow\left[=\left(\left(W \circ W_{l}\right)(G)\right) \cap[x, \rightarrow[\right.\right.$.

Let us show now that $V^{R}$ is a neighbournet, i.e. $V^{R}\{x\}=\tilde{V}\{x\}-P(x)$ is a neighbourhood of $x$, i.e. $x \notin \operatorname{cl} P(x)$. Note that $P(x) \subset] \leftarrow, x[$. Otherwise there is $y \in P(x), x<y$. If $x \notin R_{U}, y=x$; hence $\tilde{V}\{y\}=\tilde{V}\{x\}$. If $x \in R_{\alpha}$ then $x<y$ $<r_{\alpha}$ and by Lemma $1(\mathrm{c}) \tilde{V}\{y\} \subset \tilde{V}\{x\}$.

Suppose that $x \in \operatorname{cl} P(x)$. Since $P(x) \subset] \leftarrow, x]$, there exists $y \in P(x)$ with $[y, x] \subset W_{l}\{x\}, y \in R_{\alpha}$ and $x<r_{\alpha}$. Let us show that this implies $x \in R_{\alpha}$. We have $\left[x, r_{\alpha}\left[\subset\left[y, r_{\alpha}\left[\subset X_{r}-\cup_{\beta<\alpha} R_{\beta}\right.\right.\right.\right.$. We also have $\left[x, r_{\alpha}\left[=\left[y, r_{\alpha}[\cap[x, \rightarrow[\subset\right.\right.\right.$ $U_{X_{r}}^{+}\{y\} \cap\left[x, \rightarrow\left[\right.\right.$. The set $G=V_{l}\{x\} \supset[y, x]$ is a neighbourhood of $y$ in $X_{r}$. Hence $U_{X_{r}}^{+}\{y\} \cap\left[x, \rightarrow\left[\subset U(G) \cap\left[x, \rightarrow\left[=\left(W^{2}\right)(G) \cap[x, \rightarrow[\right.\right.\right.\right.$. By (ii) we have $W^{2}(G) \cap\left[x, \rightarrow\left[=\left(\left(W \circ W_{l}\right)(G)\right) \cap\left[x, \rightarrow\left[=\left(\left(W \circ W_{l}\right)\left(W_{l}\{x\}\right)\right) \cap[x, \rightarrow[\right.\right.\right.\right.$ since $G=W_{l}\{X\}$. Since $W_{l}$ is transitive, $\left(\left(W \circ W_{l}^{2}\right)\{x\}\right) \cap[x, \rightarrow[=$ $\left(\left(W \circ W_{l}\right)\{x\}\right) \cap\left[x, \rightarrow\left[=\left(W \circ W_{l}\right)_{r}\{x\}\right.\right.$. By (i) $\left(W \circ W_{l}\right)_{r}\{x\}=\left(W^{2}\right)_{r}\{x\}=$ $\left(W^{2}\right)_{r}\{x\} \subset U\{x\}$. Hence $\left[x, r_{\alpha}\left[\subset\left(X_{r}-\cup_{\beta<\alpha} R_{\beta}\right) \cap U\{x\}\right.\right.$, i.e., $x \in$ $R\left(X_{r}-\cup_{\beta<\alpha} R_{\beta}, r_{\alpha}, U\right)=R_{\alpha}$, and $\left.\tilde{V}\{x\}=\right] \leftarrow, r_{\alpha}[=\tilde{V}\{y\}$, hence $y \notin P(x)$. This contradiction proves that $x \notin \operatorname{cl} P(x)$ and hence $V^{R}$ is a neighbournet in $X$.

THEOREM 3. In a GO-space, for each neighbournet $U$ there exists a transitive neighbournet $V \subset U^{2+}$.

Proof. Suppose that each $U\{x\}$ is open in $X$. Apply Theorem 1 to the Sorgenfrey space $X_{r}$ and the neighbournet $U$ in $X_{r}$. We get a transitive neighbournet $W^{R}$ in $X_{r}$, such that $W^{R} \subset U_{X_{r}}^{+} \subset U^{+}\left(U_{X_{r}^{+}}^{+}\right.$is is the result of operation ${ }^{+}$in $X_{r}$ ). Similarly we find a transitive neighbournet $W^{L}$ in $X_{l}$ such that $W^{L} \subset U^{+}$. We may assume that $W^{R}=W_{r}^{R}$, and $W^{L}=W^{L}$ and set $W=W^{L} \cup W^{R}$. Then $W$ is a neighbournet in $X$, and $W_{r}=W^{R}$ and $W_{l}=W^{L}$ are transitive. Now apply Proposition 2 to $\tilde{U}=W^{2}$. We get a transitive neighbournet $V \subset \tilde{U}^{+}=W^{2+} \subset$ $\left(U^{+}\right)^{2+}$. To show that $\left(U^{+}\right)^{2+}=U^{2+}$, consider an open $G$ in $X$. Then $U(G)$ is open too and $\left(U^{+}\right)^{2}(G)=U^{+}\left(U^{+}(G)\right)=U^{+}(U(G))=U(U(G))=U^{2}(G)$. 
Since $U^{2+} \subset U^{3}$ we get

THEOREM 4. Each GO-space is 3 transitive.

It was explained in the Introduction that $k$-transitive $\gamma$-spaces are nonarchimedean quasimetrizable spaces; therefore we have

THEOREM 5. Each GO $\gamma$-space is nonarchimedean quasimetrizable.

IV. Remarks and examples. (i) Sorgenfrey spaces need not be 1-transitive and (ii) GO-spaces need not be 2-transitive. In this sense Theorems 1 and 3 cannot be improved.

To show (i) take the Sorgenfrey line $S$ which has the reals as the underlying set and whose topology has the intervals $[a, b[$ as a base. The neighbournet $U$ defined by $U\{x\}=\left[x, x+1\left[\right.\right.$ for each irrational $x$ and $U\left\{x_{n}\right\}=\left[x_{n}, x_{n}+1 / n[\right.$ for the $n$th rational $x_{n}$ does not contain any transitive neighbournet $V$. Otherwise, set $S_{i}=\left\{x \in S \mid\left[x, x+1 / i[\subset V\{x\}\}\right.\right.$. Some $S_{i}$ is dense in an open interval $I$. Pick a rational $x_{n} \in I$ with $U\left\{x_{n}\right\} \subset I$ and $n>i$ and let $x \in X_{i} \cap V\left\{x_{n}\right\}$; then $[x, x+$ $1 / i\left[\subset V\{x\} \subset V\left\{x_{n}\right\} \subset U\left\{x_{n}\right\} \subset\left[x_{n}, x_{n}+1 / n\right]\right.$, and hence $n<i$.

To show (ii) take the Engelking-Lutzer line $X$ which has the reals as the underlying set, and which has the intervals $[a, b[$ with rational $a$ and $] c, d]$ with irrational $d$ as a base for the topology [ELu]. Consider a neighbournet $U$ such that $U\{x\}=] x-1, x]$ for each irrational $x$ and $U\left\{x_{n}\right\} \subset\left[x_{n}, x_{n}+1 / n[\right.$ for the $n$th rational $x_{n}$ and where $U\left\{x_{n}\right\}$ is either disjoint from or contained in each of $U\left\{x_{1}\right\}, \ldots, U\left\{x_{n-1}\right\}$. Then $\left.U^{2}\left\{x_{n}\right\} \subset\right] x_{n}-1, x_{n}+1 / n\left[\right.$, and $U^{2}$ does not contain any transitive neighbournet $V$. Otherwise set $X_{i}=\{x \in X \mid[x, x+1 / i[\subset$ $V\{x\}\}, X_{0}=X-\cup_{i>1} X_{i}$, and $\left.\left.\tilde{X}_{i}=\left\{x \in X_{0} \mid\right] x-1 / i, x\right] \subset V\{x\}\right\}$. One of the sets $X_{i}, \tilde{X}_{i}$ is dense in an open interval $I$. Let us show first that it cannot be $\tilde{X}_{i}$. If $\tilde{X}_{i}$ would be dense in $I$, pick a rational $x \in I$ and let $[x, x+2 / k[\subset V\{x\}, k>i$. Now pick $y \in \tilde{X}_{i} \cap[x, x+1 / k[$. Then $y-1 / i<x<y<x+1 / k$, and $] y-$ $1 / i, y] \subset V\{y\}$ and since $V$ is transitive, $V\{x\} \subset V\{y\}$. Hence $[y, y+1 / k[\subset$ $\left[x, x+2 / k\left[\subset V\{y\}\right.\right.$, i.e., $y \in X_{k}$ and that contradicts $y \in \tilde{X}_{i}$. Therefore, some $X_{i}$ must be dense in $I$. Pick a rational $x_{n} \in I$ with $n>i$ and let $x \in X_{i} \cap\left[x_{n}, x_{n}+\right.$ $1 / n\left[\cap V\left\{x_{n}\right\}\right.$. Since $V$ is transitive, $\left[x, x+1 / i\left[\subset V\{x\} \cap\left[x_{n}, \rightarrow\left[\subset V\left\{x_{n}\right\} \cap\right.\right.\right.\right.$ $\left[x_{n}, \rightarrow\left[\subset U^{2}\left\{x_{n}\right\} \cap\left[x_{n}, \rightarrow[\subset] \leftarrow, x_{n}+1 / n\left[\cap\left[x_{n}, \rightarrow[=\right.\right.\right.\right.\right.$ $\left[x_{n}, x_{n}+1 / n\left[\right.\right.$ and hence $n \leqslant i$. Hence no set $X_{i}$ can be dense in $I$ and this contradiction completes the proof.

The author acknowledges the privilege of having seen [B] and [FL3] prior to publication. The author is indebted to David Lutzer for his help in the preparation of this paper.

\section{REFERENCES}

[B] H. R. Bennett, Quasimetrizability and the $\gamma$-space property in certain generalized ordered spaces, Topology Proceedings 4 (1979), 1-13.

[ELu] R. Engelking and D. J. Lutzer, Paracompactness in ordered spaces, Fund. Math. 94 (1976).

[FL1] P. Fletcher and W. F. Lindgren, Transitive quasiuniformities, J. Math. Anal. Appl. 39 (1972), $397-405$. 
[FL2]

[FL3] Quasiuniformities with a transitive base, Pacific J. Math. 43 (1972), 619-631.

, Quasiuniform spaces (to appear).

[F] R. Fox, On metrizability and quasi-metrizability (to appear).

[G] G. Gruenhage, A note on quasi-metrizability, Canad. J. Math. 29 (1977), 360-366.

[H] R. E. Hodel, Spaces defined by sequences of open covers which guarantee that certain sequences have cluster points, Duke Math. J. 39 (1972), 253-263.

[J1] H. Junnila, Covering properties and quasi-uniformities of topological spaces, Ph.D. thesis, Virginia Polytech. Inst. and State Univ., 1978.

[J2] __ Neighbournets, Pacific J. Math. 76 (1978), 83-108.

[K] J. Kofner, On $\Delta$-metrizable spaces, Math. Notes 13 (1973), 168-174.

[LF] W. F. Lindgren and P. Fletcher, Locally quasi-uniform spaces with countable bases, Duke Math. J. 41 (1974), 231-240.

[Lu] D. J. Lutzer, On generalized ordered spaces, Dissertationes Math. 89 (1971).

[N1] V. V. Niemytzki, On the third axiom of metric spaces, Trans. Amer. Math. Soc. 29 (1927), 507-513.

[N2] Über die Axioms des metrischen Raumes, Math. Ann. 106 (1931), 666-671.

[T] Topology Proceedings 2 (1977), p. 687.

[W] W. A. Wilson, On quasi-metric spaces, Amer. J. Math. 53 (1931), 675-684.

Department of Mathematics, Texas Tech University, Lubbock, TeXas 79409

Current address: Department of Mathematics, George Mason University, Fairfax, Virginia 22030 